\title{
A note on type 2 degenerate poly-Cauchy polynomials and numbers of
} the second kind

\section{Waseem A. Khan}

Department of Mathematics and Natural Sciences, Prince Mohammad Bin Fahd University, P.O Box 1664, Al Khobar 31952, Kingdom of Saudi Arabia

$$
\text { E-mail: wkhan1@pmu.edu.sa }
$$

\begin{abstract}
Kim-Kim [] studied the type 2 degenerate poly-Bernoulli numbers and polynomials by using modified degenerate polylogarithm function. In this paper, we construct the type 2 degenerate poly-Cauchy polynomials and numbers of the second kind, called degenerate poly-Cauchy polynomials and numbers of the second kind by using degenerate polylogarithm function and derive several properties on the degenerate poly-Cauchy polynomials and numbers of the second kind. Furthermore, we consider the degenerate unipoly-Cauchy polynomials of the second kind and discuss some properties of them.
\end{abstract}

Keywords: Degenerate polylogarithm functions, Degenerate poly-Cauchy polynomials, Degenerate unipoly-Cauchy polynomials.

2010 Mathematics Subject Classification: 11B68, 11B83, 11S80.

\section{Introduction}

In recent years, many researchers have been studied for various degenerate versions of many special polynomials and numbers which included the degenerate Stirlings numbers of the second kind, degenerate central factorial numbers of the second kind, degenerate Bernoulli numbers of the second kind, degenerate Bernstein polynomials, degenerate Bell numbers and polynomials, degenerate central Bell numbers and polynomials, degenerate complete Bell polynomials and numbers, degenerate Cauchy numbers, and others. (see []). Carlitz [] initiated a study of degenerate versions of some special polynomials and numbers, namely the degenerate Bernoulli and Euler polynomials and numbers.

As is well known, the Cauchy polynomials $C_{n}(x)$ are defined by the means of the following generating function

$$
\int_{0}^{1}(1+t)^{x+y} d y=\frac{t}{\log (1+t)}(1+t)^{x}=\sum_{n=0}^{\infty} C_{n}(x) \frac{t^{n}}{n !},(\text { see }[1,14,15,19]) .
$$

In case when $x=0, C_{n}=C_{n}(0)$ are called the Cauchy numbers.

The higher-order Bernoulli polynomials are defined by

$$
\left(\frac{t}{e^{t}-1}\right)^{\alpha} e^{x t}=\sum_{n=0}^{\infty} B_{n}^{(\alpha)}(x) \frac{t^{n}}{n !}, \text { see []). }
$$

For $x=0, B_{n}^{(\alpha)}=B_{n}^{(\alpha)}(0)$ are called the higher-order Bernoulli numbers and $B_{n}(x)=$ $B_{n}^{(1}(x)$ are called the ordinary Bernoulli polynomials. 
From (1.1) and (1.2), we note that

$$
C_{n}(x)=B_{n}^{(n)}(x+1),(n \geq 0),(\text { see []). }
$$

For $\lambda \in \mathbb{R}$, the degenerate exponential functions are defined as

$$
e_{\lambda}^{x}(t)=(1+\lambda t)^{\frac{x}{\lambda}}, e_{\lambda}(t) e_{\lambda}^{1}(t)=(1+\lambda t)^{\frac{1}{\lambda}},(\text { see }[10-15]) .
$$

Here we note that

$$
e_{\lambda}^{x}(t)=\sum_{n=0}^{\infty}(x)_{n, \lambda} \frac{t^{n}}{n !}
$$

where $(x)_{0, \lambda}=1,(x)_{n, \lambda}=x(x-\lambda)(x-2 \lambda) \cdots(x-(n-1) \lambda),(n \geq 1)$.

In $[1,2]$, Carlitz considered the degenerate Bernoulli polynomials which are given by

$$
\frac{t}{e_{\lambda}(t)-1} e_{\lambda}^{x}(t)=\frac{t}{(1+\lambda t)^{\frac{1}{\lambda}}-1}(1+\lambda t)^{\frac{x}{\lambda}}=\sum_{n=0}^{\infty} \beta_{n, \lambda}(x) \frac{t^{n}}{n !} .
$$

On setting $x=0, \beta_{n, \lambda}=\beta_{n, \lambda}(0)$ are called degenerate Bernoulli numbers.

For $k \in \mathbb{Z}$, the degenerate modified polyexponential function [16] is defined by KimKim to be

$$
\operatorname{Ei}_{k, \lambda}(x)=\sum_{n=1}^{\infty} \frac{(1)_{n, \lambda} x^{n}}{(n-1) ! n^{k}},(|x|<1)
$$

Note that

$$
\operatorname{Ei}_{1, \lambda}(x)=\sum_{n=1}^{\infty} \frac{(1)_{n, \lambda} x^{n}}{n !}=e_{\lambda}(x)-1 .
$$

In [16], Kim-Kim considered the type 2 degenerate poly-Bernoulli polynomials are defined by means of the following generating function

$$
\frac{\operatorname{Ei}_{k, \lambda}\left(\log _{\lambda}(1+t)\right)}{e_{\lambda}(t)-1} e_{\lambda}^{x}(t)=\sum_{n=0}^{\infty} B_{n, \lambda}^{(k)}(x) \frac{t^{n}}{n !},(k \in \mathbb{Z}) .
$$

In case when $x=0, B_{n, \lambda}^{(k)}=B_{n, \lambda}^{(k)}(0)$ are called the type 2 degenerate poly-Bernoulli numbers.

Kim [14] defined the degenerate Cauchy polynomials $C_{n, \lambda}(x)$ as follows:

$$
\begin{gathered}
\int_{0}^{1}\left(1+\log (1+\lambda t)^{\frac{1}{\lambda}}\right)^{x+y} d y=\frac{\frac{1}{\lambda}(\log (1+\lambda t))}{\log \left(1+\frac{1}{\lambda} \log (1+\lambda t)\right)}\left(1+\log (1+\lambda t)^{\frac{1}{\lambda}}\right)^{x} \\
=\sum_{n=0}^{\infty} C_{n, \lambda}^{*}(x) \frac{t^{n}}{n !},(\text { see }[2,7,18,21])
\end{gathered}
$$

Letting $x=0, C_{n, \lambda}=C_{n, \lambda}(0)$ are the degenerate Cauchy numbers.

The degenerate Cauchy polynomials $C_{n, \lambda}(x)$ of the second kind are introduced in [14] as follows

$$
\frac{t}{\log \left(1+\frac{1}{\lambda} \log (1+\lambda t)\right)}\left(1+\frac{1}{\lambda} \log (1+\lambda t)\right)^{x}=\sum_{n=0}^{\infty} C_{n, \lambda}(x) \frac{t^{n}}{n !},(\text { see }[9,10,24]) .
$$

In case when $x=0, C_{n, \lambda}=C_{n \lambda}(0)$ are called the degenerate Cauchy polynomials of the second kind. 
In [22], the degenerate Daehee polynomials $D_{n, \lambda}(x)$ are defined by

$$
\frac{\log _{\lambda}(1+t)}{t}(1+t)^{x}=\sum_{n=0}^{\infty} D_{n, \lambda}(x) \frac{t^{n}}{n !}, \text { (see []). }
$$

For $x=0, D_{n, \lambda}=D_{n, \lambda}(0)$ are called the degenerate Daehee numbers.

The degenerate Stirling numbers of the first kind are defined by

$$
\frac{1}{k !}\left(\log _{\lambda}(1+t)\right)^{k}=\sum_{n=k}^{\infty} S_{1, \lambda}(n, k) \frac{t^{n}}{n !}, \quad(k \geq 0),(\text { see }[10,16,18]) .
$$

Note here that $\lim _{\lambda \rightarrow 0} S_{1, \lambda}(n, k)=S_{1}(n, k)$, where $S_{1}(n, k)$ are the Stirling numbers of the first kind given by

$$
\frac{1}{k !}(\log (1+t))^{k}=\sum_{n=k}^{\infty} S_{1}(n, k) \frac{t^{n}}{n !},(k \geq 0),(\text { see }[7,11]) .
$$

The degenerate Stirling numbers of the second kind are given by

$$
\frac{1}{k !}\left(e_{\lambda}(t)-1\right)^{k}=\sum_{n=l}^{\infty} S_{2, \lambda}(n, l) \frac{t^{n}}{n !},(\text { see }[9]) .
$$

Observe here that $\lim _{\lambda \rightarrow 0} S_{2, \lambda}(n, k)=S_{1}(n, k)$, where $S_{2}(n, k)$ are the Stirling numbers of the second kind given by

$$
\frac{1}{k !}\left(e^{t}-1\right)^{k}=\sum_{n=l}^{\infty} S_{2}(n, l) \frac{t^{n}}{n !},(\text { see }[1-22]) .
$$

By the motivation of the works of Kim-Kim [], we first define the type 2 degenerate poly-Cauchy polynomials of the second kind by using the degenerate polyexponential functions. We investigate some new properties of these numbers and polynomials and derive some new identities and relations between the new type of degenerate poly-Cauchy polynomials of the second kind. Furthermore, we consider the type 2 degenerate unipoly-Cauchy polynomials of the second kind and discuss some identities of them.

\section{Type 2 degenerate poly-Cauchy polynomials of the second kind}

For $k \in \mathbb{Z}$, by using equations (1.9) and (1.11), we define the type 2 degenerate poly-Cauchy polynomials of the second kind as follows:

$$
\frac{\operatorname{Ei}_{\mathrm{k}, \lambda}\left(\log _{\lambda}(1+\mathrm{t})\right)}{\log \left(1+\frac{1}{\lambda} \log (1+\lambda t)\right)}\left(1+\frac{1}{\lambda} \log (1+\lambda t)\right)^{x}=\sum_{n=0}^{\infty} C_{n, \lambda}^{(k)}(x) \frac{t^{n}}{n !}
$$

In case when $x=0$ in $(2.1), C_{n, \lambda}^{(k)}=C_{n, \lambda}^{(k)}(0)$ are called the type 2 degenerate polyCauchy numbers of the second kind. For $k=1$, by $(2.1)$, we note that

$$
C_{n, \lambda}^{(1)}=C_{n, \lambda},(n \in \mathbb{N} \cup\{0\})
$$

are called the degenerate Cauchy numbers of the second kind.

By using equations (1.7), (1.11) and (2.1), we observe that

$$
\sum_{n=0}^{\infty} C_{n, \lambda}^{(k)} \frac{t^{n}}{n !}=\frac{\operatorname{Ei}_{\mathrm{k}, \lambda}\left(\log _{\lambda}(1+\mathrm{t})\right)}{\log \left(1+\frac{1}{\lambda} \log (1+\lambda t)\right)}
$$




$$
\begin{gathered}
=\frac{t}{\log \left(1+\frac{1}{\lambda} \log (1+\lambda t)\right)} \frac{1}{t} \sum_{m=1}^{\infty} \frac{\left(\log _{\lambda}(1+t)\right)^{m}}{(m-1) ! m^{k}} \\
=\frac{t}{\log \left(1+\frac{1}{\lambda} \log (1+\lambda t)\right)} \frac{1}{t} \sum_{m=0}^{\infty} \frac{\left(\log _{\lambda}(1+t)\right)^{m+1}}{(m+1) !(m+1)^{k-1}} \\
=\frac{t}{\log \left(1+\frac{1}{\lambda} \log (1+\lambda t)\right)} \frac{1}{t} \sum_{m=0}^{\infty} \frac{1}{(m+1)^{k-1}} \sum_{l=m+1}^{\infty} S_{1, \lambda}(l, m+1) \frac{t^{l}}{l !} \\
=\frac{t}{\log \left(1+\frac{1}{\lambda} \log (1+\lambda t)\right)} \frac{1}{t} \sum_{m=0}^{\infty} \frac{1}{(m+1)^{k-1}} \sum_{l=m}^{\infty} S_{1, \lambda}(l+1, m+1) \frac{t^{l}}{(l+1) !} \\
=\left(\sum_{s=0}^{\infty} C_{s, \lambda} \frac{t^{s}}{s !}\right)\left(\sum_{l=0}^{\infty} \sum_{m=0}^{l} \frac{1}{(m+1)^{k-1}} \frac{S_{1, \lambda}(l+1, m+1)}{l+1} \frac{t^{l}}{l !}\right) \\
L . H . S=\sum_{n=0}^{\infty}\left(\sum_{l=0}^{n} \sum_{m=0}^{l}\left(\begin{array}{l}
n \\
l
\end{array}\right) C_{n-l, \lambda} \frac{S_{1, \lambda}(l+1, m+1)}{l+1(m+1)^{k-1}}\right) \frac{t^{n}}{n !} .
\end{gathered}
$$

Therefore, by (2.3), we obtain the following theorem.

Theorem 2.1. For $n \geq 0$ and $k \in \mathbb{Z}$, we have

$$
C_{n, \lambda}^{(k)}=\sum_{l=0}^{n} \sum_{m=0}^{l}\left(\begin{array}{l}
n \\
l
\end{array}\right) C_{n-l, \lambda} \frac{S_{1, \lambda}(l+1, m+1)}{l+1(m+1)^{k-1}} .
$$

Corollary 2.1. For $n \geq 0$ and $k \in \mathbb{Z}$, we have

$$
C_{n, \lambda}^{(1)}=\sum_{l=0}^{n} \sum_{m=0}^{l}\left(\begin{array}{l}
n \\
l
\end{array}\right) C_{n-l, \lambda} \frac{S_{1, \lambda}(l+1, m+1)}{l+1} .
$$

Moreover,

$$
\sum_{l=0}^{n} \sum_{m=0}^{l}\left(\begin{array}{l}
n \\
l
\end{array}\right) C_{n-l, \lambda} \frac{S_{1, \lambda}(l+1, m+1)}{l+1}=0 .
$$

From (2.1), we observe that

$$
\begin{gathered}
\sum_{n=0}^{\infty} C_{n, \lambda}^{(k)}(x) \frac{t^{n}}{n !} \\
=\frac{\operatorname{Ei}_{\mathrm{k}, \lambda}\left(\log _{\lambda}(1+\mathrm{t})\right)}{\log \left(1+\frac{1}{\lambda} \log (1+\lambda t)\right)}\left(1+\frac{1}{\lambda} \log (1+\lambda t)\right)^{x} \\
=\left(\sum_{l=0}^{\infty} C_{l, \lambda}^{(k)} \frac{t^{l}}{l !}\right)\left(\sum_{m=0}^{\infty}\left(\begin{array}{c}
x \\
m
\end{array}\right)\left(\frac{1}{\lambda} \log (1+\lambda t)\right)^{m}\right) \\
=\left(\sum_{l=0}^{\infty} C_{l, \lambda}^{(k)} \frac{t^{l}}{l !}\right)\left(\sum_{m=0}^{\infty}(x)_{m} \lambda^{-m} \sum_{s=m}^{\infty} S_{1}(s, m) \lambda^{s} \frac{t^{s}}{s !}\right) \\
=\left(\sum_{l=0}^{\infty} C_{l, \lambda}^{(k)} \frac{t^{l}}{l !}\right)\left(\sum_{s=0}^{\infty} \sum_{m=0}^{s}(x)_{m} \lambda^{-m} S_{1}(s, m) \lambda^{s} \frac{t^{s}}{s !}\right) \\
\text { R.H.S }=\sum_{n=0}^{\infty}\left(\sum_{l=0}^{n} \sum_{m=0}^{n-l}\left(\begin{array}{c}
n \\
l
\end{array}\right) C_{l, \lambda}^{(k)}(x)_{m} \lambda^{n-l-m} S_{1}(n-l, m)\right) \frac{t^{n}}{n !} .
\end{gathered}
$$

By comparing the coefficients on both sides of (2.6), we obtain the following theorem.

Theorem 2.2. Let $n \geq 0$ and $k \in \mathbb{Z}$. Then we have 


$$
C_{n, \lambda}^{(k)}(x)=\sum_{l=0}^{n} \sum_{m=0}^{n-l}\left(\begin{array}{l}
n \\
l
\end{array}\right) C_{l, \lambda}^{(k)}(x)_{m} \lambda^{n-l-m} S_{1}(n-l, m) .
$$

In [ ], it is well known that the degenerate Bernoulli polynomials of the second kind are defined by

$$
\frac{t}{\log _{\lambda}(1+t)}(1+t)^{x}=\sum_{n=0}^{\infty} b_{n, \lambda}(x) \frac{t^{n}}{n !} .
$$

For $x=0, b_{n, \lambda}=b_{n, \lambda}(0)$ are called degenerate Bernoulli numbers of the second kind.

From (1.7), we note that

$$
\begin{gathered}
\frac{d}{d x} \operatorname{Ei}_{\mathrm{k}, \lambda}\left(\log _{\lambda}(1+\mathrm{x})\right)=\frac{\mathrm{d}}{\mathrm{dx}} \sum_{\mathrm{n}=1}^{\infty} \frac{(1)_{\mathrm{n}, \lambda}\left(\log _{\lambda}(1+\mathrm{x})\right)^{\mathrm{n}}}{(\mathrm{n}-1) ! \mathrm{n}^{\mathrm{k}}} \\
=\frac{(1+x)^{\lambda-1}}{\log _{\lambda}(1+x)} \sum_{n=1}^{\infty} \frac{(1)_{n, \lambda}\left(\log _{\lambda}(1+x)\right)^{n}}{(n-1) ! n^{k-1}}=\frac{(1+x)^{\lambda-1}}{\log _{\lambda}(1+x)} \operatorname{Ei}_{\mathrm{k}-1, \lambda}\left(\log _{\lambda}(1+x)\right) .
\end{gathered}
$$

Thus, from (2.1) and (2.9), we have

$$
\begin{aligned}
& \sum_{n=0}^{\infty} C_{n, \lambda}^{(k)} \frac{x^{n}}{n !}=\frac{1}{\log \left(1+\frac{1}{\lambda} \log (1+\lambda x)\right)} \operatorname{Ei}_{k, \lambda}\left(\log _{\lambda}(1+x)\right)
\end{aligned}
$$

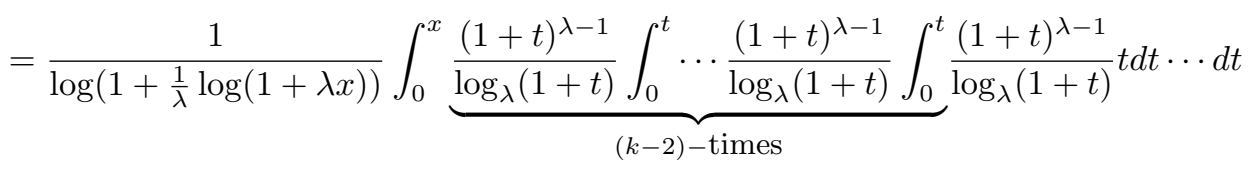

$$
\begin{aligned}
& =\frac{x}{\log \left(1+\frac{1}{\lambda} \log (1+\lambda x)\right)} \sum_{m=0}^{\infty} \sum_{m_{1}+\cdots+m_{k-1}=m}\left(\begin{array}{c}
m \\
m_{1}+\cdots+m_{k-1}
\end{array}\right) \\
& \times \frac{b_{m_{1}, \lambda}(\lambda-1)}{m_{1}+1} \frac{b_{m_{2}, \lambda}(\lambda-1)}{m_{1}+m_{2}+1} \cdots \frac{b_{m_{k-1}, \lambda}(\lambda-1)}{m_{1}+\cdots+m_{k-1}+1} \frac{x^{m}}{m !} \\
& \sum_{n=0}^{\infty} C_{n, \lambda}^{(k)} \frac{x^{n}}{n !}=\sum_{n=0}^{\infty} \sum_{m=0}^{n}\left(\begin{array}{c}
n \\
m
\end{array}\right) \sum_{m_{1}+\cdots+m_{k-1}=m}\left(\begin{array}{c}
m \\
m_{1}+\cdots+m_{k-1}
\end{array}\right) \\
& \times \frac{b_{m_{1}, \lambda}(\lambda-1)}{m_{1}+1} \frac{b_{m_{2}, \lambda}(\lambda-1)}{m_{1}+m_{2}+1} \cdots \frac{b_{m_{k-1}, \lambda}(\lambda-1)}{m_{1}+\cdots+m_{k-1}+1} C_{n-m, \lambda} \frac{x^{n}}{n !} .
\end{aligned}
$$

Therefore, by (2.10), we obtain the following theorem.

Theorem 2.3. For $n \geq 0$, we have

$$
\begin{gathered}
C_{n, \lambda}^{(k)}=\sum_{m=0}^{n}\left(\begin{array}{c}
n \\
m
\end{array}\right) \sum_{m_{1}+\cdots+m_{k-1}=m}\left(\begin{array}{c}
m \\
m_{1}+\cdots+m_{k-1}
\end{array}\right) \\
\times \frac{b_{m_{1}, \lambda}(\lambda-1)}{m_{1}+1} \frac{b_{m_{2}, \lambda}(\lambda-1)}{m_{1}+m_{2}+1} \cdots \frac{b_{m_{k-1}, \lambda}(\lambda-1)}{m_{1}+\cdots+m_{k-1}+1} C_{n-m, \lambda} .
\end{gathered}
$$

Corollary 2.2. For $k \geq 2$, we have

$$
C_{n, \lambda}^{(2)}=\sum_{m=0}^{n}\left(\begin{array}{c}
n \\
m
\end{array}\right) \frac{b_{m, \lambda}(\lambda-1)}{m+1} C_{n-m, \lambda} .
$$

Let $k \geq 1$, be an integer. For $s \in \mathbb{C}$, we define the function $\eta_{k, \lambda}(s)$ as

$$
\eta_{k, \lambda}(s)=\frac{1}{\Gamma(s)} \int_{0}^{\infty} \frac{t^{s-1}}{\log \left(1+\frac{1}{\lambda} \log (1+\lambda t)\right)} \operatorname{Ei}_{k, \lambda}\left(\log _{\lambda}(1+t)\right) d t
$$




$$
\begin{aligned}
& =\frac{1}{\Gamma(s)} \int_{0}^{1} \frac{t^{s-1}}{\log \left(1+\frac{1}{\lambda} \log (1+\lambda t)\right)} \operatorname{Ei}_{k, \lambda}\left(\log _{\lambda}(1+t)\right) d t \\
& +\frac{1}{\Gamma(s)} \int_{1}^{\infty} \frac{t^{s-1}}{\log \left(1+\frac{1}{\lambda} \log (1+\lambda t)\right)} \operatorname{Ei}_{k, \lambda}\left(\log _{\lambda}(1+t)\right) d t
\end{aligned}
$$

The second integral converges absolutely for any $s \in \mathbb{C}$ and hence, the second term on the right hand side vanishes at non-positive integers. That is,

$$
\lim _{s \rightarrow-m}\left|\frac{1}{\Gamma(s)} \int_{1}^{\infty} \frac{t^{s-1}}{\log \left(1+\frac{1}{\lambda} \log (1+\lambda t)\right)} \operatorname{Ei}_{k, \lambda}\left(\log _{\lambda}(1+t)\right) d t\right| \leq \frac{1}{\Gamma(-m)} M=0 .
$$

On the other hand, for $\Re(s)>0$, the first integral in (2.13) can be written as

$$
\frac{1}{\Gamma(s)} \sum_{l=0}^{\infty} \frac{C_{l, \lambda}^{(k)}}{l !} \frac{1}{s+l}
$$

which defines an entire function of $s$. Thus, we may include that $\eta_{k, \lambda}(s)$ can be continued to an entire function of $s$.

Further, from (2.12) and (2.13), we obtain

$$
\begin{gathered}
\eta_{k, \lambda}(-m)=\lim _{s \rightarrow-m} \frac{1}{\Gamma(s)} \int_{0}^{1} \frac{t^{s-1}}{\log \left(1+\frac{1}{\lambda} \log (1+\lambda t)\right)} \operatorname{Ei}_{k, \lambda}\left(\log _{\lambda}(1+t)\right) d t \\
=\lim _{s \rightarrow-m} \frac{1}{\Gamma(s)} \int_{0}^{1} t^{s-1} \sum_{l=0}^{\infty} \frac{C_{l, \lambda}^{(k)} t^{l}}{l !} d t=\lim _{s \rightarrow-m} \frac{1}{\Gamma(s)} \sum_{l=0}^{\infty} \frac{C_{l, \lambda}^{(k)}}{s+l} \frac{1}{l !} \\
=\cdots+0+\cdots+0+\lim _{s \rightarrow-m} \frac{1}{\Gamma(s)} \frac{1}{s+m} \frac{C_{m, \lambda}^{(k)}}{m !}+0+0+\cdots \\
=\lim _{s \rightarrow-m} \frac{\left(\frac{\Gamma(1-s) \sin \pi s}{\pi}\right)}{s+m} \frac{C_{m, \lambda}^{(k)}}{m !}=\Gamma(1+m) \cos (\pi m) \frac{C_{m, \lambda}^{(k)}}{m !} \\
=(-1)^{m} C_{m, \lambda, 2}^{(k)} .
\end{gathered}
$$

Therefore, by (2.14), we obtain the following theorem.

Theorem 2.4. Let $k \geq 1$ and $m \in \mathbb{N} \bigcup\{0\}, s \in \mathbb{C}$, we have

$$
\eta_{k, \lambda}(-m)=(-1)^{m} C_{m, \lambda}^{(k)}
$$

Replacing $t$ by $\frac{1}{\lambda} e_{\lambda}(t)-1$ in (2.1) and using (1.1), we get

Now

$$
\sum_{m=0}^{\infty} C_{m, \lambda}^{(k)}(x) \lambda^{-m} \frac{\left(e_{\lambda}(t)-1\right)^{m}}{m !}=\frac{\operatorname{Ei}_{\mathrm{k}, \lambda}\left(\frac{\mathrm{t}}{\lambda}\right)}{\log (1+t)}(1+t)^{x}
$$

$$
\begin{gathered}
\frac{\operatorname{Ei}_{\mathrm{k}, \lambda}\left(\frac{\mathrm{t}}{\lambda}\right)}{\log (1+t)}(1+t)^{x}=\left(\frac{t}{\log (1+t)}(1+t)^{x}\right)\left(\frac{\operatorname{Ei}_{\mathrm{k}, \lambda}\left(\frac{\mathrm{t}}{\lambda}\right)}{t}\right) \\
=\left(\sum_{n=0}^{\infty} C_{n}(x) \frac{t^{n}}{n !}\right)\left(\frac{1}{t} \sum_{m=1}^{\infty} \frac{\lambda^{-m}(1)_{m, \lambda} t^{m}}{(m-1) ! m^{k}}\right) \\
=\left(\sum_{n=0}^{\infty} C_{n}(x) \frac{t^{n}}{n !}\right)\left(\sum_{m=0}^{\infty} \frac{\lambda^{-m-1}(1)_{m+1, \lambda} t^{m}}{m !(m+1)^{k}}\right) \\
\text { L.H.S }=\sum_{n=0}^{\infty}\left(\sum_{m=0}^{n}\left(\begin{array}{c}
n \\
m
\end{array}\right) C_{n-m}(x) \frac{\lambda^{-m-1}(1)_{m+1, \lambda}}{(m+1)^{k}}\right) \frac{t^{n}}{n !} .
\end{gathered}
$$


On the other hand,

$$
\begin{gathered}
\sum_{m=0}^{\infty} C_{m, \lambda}^{(k)}(x) \lambda^{-m} \frac{\left(e_{\lambda}(t)-1\right)^{m}}{m !}=\sum_{m=0}^{\infty} C_{m, \lambda}^{(k)}(x) \lambda^{-m} \sum_{n=m}^{\infty} S_{2}(n, m) \lambda^{n} \frac{t^{n}}{n !} \\
=\sum_{n=0}^{\infty}\left(\sum_{m=0}^{n} \lambda^{n-m} C_{m, \lambda}^{(k)}(x) S_{2}(n, m)\right) \frac{t^{n}}{n !} .
\end{gathered}
$$

Therefore, by equations (2.15) and (2.16), we obtain the following theorem.

Theorem 2.5. Let $k \geq 1$ and $m \in \mathbb{N} \bigcup\{0\}, s \in \mathbb{C}$, we have

$$
\begin{gathered}
\sum_{m=0}^{n}\left(\begin{array}{c}
n \\
m
\end{array}\right) C_{n-m}(x) \frac{\lambda^{-m-1}(1)_{m+1, \lambda}}{(m+1)^{k}} \\
=\sum_{m=0}^{n} \lambda^{n-m} C_{m, \lambda}^{(k)}(x) S_{2}(n, m) .
\end{gathered}
$$

From (2.1), we note that

$$
\operatorname{Ei}_{k, \lambda}\left(\log _{\lambda}(1+t)\right)=\left(\sum_{n=0}^{\infty} C_{n, \lambda}^{(k)} \frac{t^{n}}{n !}\right)\left(\log \left(1+\frac{1}{\lambda} \log (1+\lambda t)\right)\right)
$$

Now

$$
\begin{gathered}
\left(\sum_{n=0}^{\infty} C_{n, \lambda}^{(k)} \frac{t^{n}}{n !}\right)\left(\log \left(1+\frac{1}{\lambda} \log (1+\lambda t)\right)\right) \\
=\left(\sum_{n=0}^{\infty} C_{n, \lambda}^{(k)} \frac{t^{n}}{n !}\right)\left(\sum_{l=1}^{\infty} \frac{\left.(-1)^{l-1} \lambda^{-l}(\log (1+\lambda t))^{l}\right)}{l}\right. \\
=\left(\sum_{n=0}^{\infty} C_{n, \lambda}^{(k)} \frac{t^{n}}{n !}\right)\left((l-1) !(-1)^{l-1} \lambda^{-l} \sum_{m=l}^{\infty} S_{1}(m, l) \lambda^{m} \frac{t^{m}}{m !}\right) \\
=\left(\sum_{n=0}^{\infty} C_{n, \lambda}^{(k)} \frac{t^{n}}{n !}\right)\left(\sum_{m=1}^{\infty}\left(\sum_{l=1}^{m}(l-1) !(-1)^{l-1} \lambda^{m-l} S_{1}(m, l)\right) \frac{t^{m}}{m !}\right) \\
\text { L.H.S }=\sum_{n=1}^{\infty}\left(\sum_{m=1}^{n} \sum_{l=1}^{m}\left(\begin{array}{c}
n \\
m
\end{array}\right) C_{n-m, \lambda}^{(k)}(l-1) !(-1)^{l-1} \lambda^{m-l} S_{1}(m, l)\right) \frac{t^{n}}{n !} .
\end{gathered}
$$

On the other hand,

$$
\begin{gathered}
\operatorname{Ei}_{k, \lambda}\left(\log _{\lambda}(1+t)\right)=\sum_{m=1}^{\infty} \frac{(1)_{m, \lambda}\left(\log _{\lambda}(1+t)\right)^{m}}{(m-1) ! m^{k}} \\
=\sum_{m=1}^{\infty} \frac{(1)_{m, \lambda}\left(\log _{\lambda}(1+t)\right)^{m}}{(m-1) ! m^{k}} \frac{m !}{m !} \\
=\sum_{m=1}^{\infty} \frac{(1)_{m, \lambda}}{m^{k-1}} \sum_{n=m}^{\infty} S_{1, \lambda}(n, m) \frac{t^{n}}{n !} \\
\text { L.H.S }=\sum_{n=1}^{\infty}\left(\sum_{m=1}^{n} \frac{(1)_{m, \lambda} S_{1, \lambda}(n, m)}{m^{k-1}}\right) \frac{t^{n}}{n !} .
\end{gathered}
$$

Therefore, by (2.17) and (2.18), we obtain the following theorem. 
Theorem 2.6. Let $k \geq 1$ and $m \in \mathbb{N} \bigcup\{0\}, s \in \mathbb{C}$, we have

$$
\begin{gathered}
\sum_{m=1}^{n} \frac{(1)_{m, \lambda} S_{1, \lambda}(n, m)}{m^{k-1}} \\
=\sum_{m=1}^{n} \sum_{l=1}^{m}\left(\begin{array}{c}
n \\
m
\end{array}\right) C_{n-m, \lambda}^{(k)}(l-1) !(-1)^{l-1} \lambda^{m-l} S_{1}(m, l) .
\end{gathered}
$$

For $k=1$ in Theorem 2.6., we get the following corollary

Corollary 2.3. For $m \in \mathbb{N} \bigcup\{0\}, s \in \mathbb{C}$, we have

$$
\begin{gathered}
\sum_{m=1}^{n}(1)_{m, \lambda} S_{1, \lambda}(n, m) \\
=\sum_{m=1}^{n} \sum_{l=1}^{m}\left(\begin{array}{c}
n \\
m
\end{array}\right) C_{n-m, \lambda}^{(1)}(l-1) !(-1)^{l-1} \lambda^{m-l} S_{1}(m, l) .
\end{gathered}
$$

Corollary 2.4. For $m \in \mathbb{N} \bigcup\{0\}, s \in \mathbb{C}$, we have

$$
C_{0, \lambda}=\sum_{m=1}^{n} \sum_{l=1}^{m}\left(\begin{array}{c}
n \\
m
\end{array}\right) C_{n-m, \lambda}^{(1)}(l-1) !(-1)^{l-1} \lambda^{m-l} S_{1}(m, l)=\left\{\begin{array}{cc}
1, & \text { if } n=0 \\
0, & \text { if } n>1 .
\end{array}\right.
$$

Now, we observe that

$$
\begin{aligned}
\frac{\operatorname{Ei}_{\mathrm{k}, \lambda}\left(\log _{\lambda}(1+\mathrm{t})\right)}{\log \left(1+\frac{1}{\lambda} \log (1+\lambda t)\right)}=\left(\frac{\lambda t}{\log (1+\lambda t)}\right)\left(\frac{\operatorname{Ei}_{\mathrm{k}, \lambda}\left(\log _{\lambda}(1+\mathrm{t})\right)}{t}\right)\left(\frac{\frac{1}{\lambda} \log (1+\lambda t)}{\log \left(1+\frac{1}{\lambda} \log (1+\lambda t)\right.}\right) \\
=\left(\sum_{n=0}^{\infty} \lambda^{l} B_{n}^{(n)} \frac{t^{n}}{n !}\right)\left(\frac{1}{t} \sum_{m=1}^{\infty} \frac{(1)_{m, \lambda}\left(\log _{\lambda}(1+t)\right)^{m}}{(m-1) ! m^{k}}\right)\left(\sum_{l=0}^{\infty} C_{l, \lambda}^{*} \frac{t^{l}}{l !}\right) \\
=\left(\sum_{n=0}^{\infty} \lambda^{l} B_{n}^{(n)} \frac{t^{n}}{n !}\right)\left(\sum_{m=0}^{\infty} \frac{(1)_{m+1, \lambda}\left(\log _{\lambda}(1+t)\right)^{m+1}}{m !(m+1)^{k}} \frac{(m+1) !}{(m+1) !}\right)\left(\sum_{l=0}^{\infty} C_{l, \lambda}^{*} \frac{t^{l}}{l !}\right) \\
=\left(\sum_{n=0}^{\infty} \lambda^{l} B_{n}^{(n)} \frac{t^{n}}{n !}\right)\left(\sum_{m=0}^{\infty} \frac{(1)_{m+1, \lambda}}{(m+1)^{k-1}} \sum_{r=m+1}^{\infty} S_{1, \lambda}(r, m+1) \frac{t^{r}}{r !}\right)\left(\sum_{l=0}^{\infty} C_{l, \lambda}^{*} \frac{t^{l}}{l !}\right) \\
=\left(\sum_{n=0}^{\infty} \lambda^{n} B_{n}^{(n)} \frac{t^{n}}{n !}\right)\left(\sum_{r=0}^{\infty} \sum_{m=0}^{r} \frac{(1)_{m+1, \lambda} S_{1, \lambda}(r+1, m+1)}{(r+1)(m+1)^{k-1}} \frac{t^{r}}{r !}\right)\left(\sum_{l=0}^{\infty} C_{l, \lambda}^{*} \frac{t^{l}}{l !}\right) \\
=\left(\sum_{n=0}^{\infty} \lambda^{n} B_{n}^{(n)} \frac{t^{n}}{n !}\right)\left(\sum_{l=0}^{\infty} \sum_{r=0}^{l}\left(\begin{array}{l}
l \\
r
\end{array}\right) \sum_{m=0}^{r} \frac{(1)_{m+1, \lambda} S_{1, \lambda}(r+1, m+1)}{(r+1)(m+1)^{k-1}} C_{l-r, \lambda}^{*}\right) \frac{t^{l}}{l !} \\
\text { R.H.S }=\sum_{n=0}^{\infty}\left(\sum_{l=0}^{n}\left(\begin{array}{l}
n \\
l
\end{array}\right) \sum_{r=0}^{l}\left(\begin{array}{l}
l \\
r
\end{array}\right) \sum_{m=0}^{r} \frac{(1)_{m+1, \lambda} S_{1, \lambda}(r+1, m+1)}{(r+1)(m+1)^{k-1}} C_{l-r, \lambda}^{*} \lambda^{n-l} B_{n-m}^{(n-m)}\right)
\end{aligned}
$$

From (2.1), we note that

$$
\frac{\operatorname{Ei}_{\mathrm{k}, \lambda}\left(\log _{\lambda}(1+\mathrm{t})\right)}{\log \left(1+\frac{1}{\lambda} \log (1+\lambda t)\right)}=\sum_{n=0}^{\infty} C_{n, \lambda}^{(k)} \frac{t^{n}}{n !} .
$$

Therefore, by (2.19) and (2.20), we obtain the following theorem. 
Theorem 2.7. Let $k \geq 1$ and $m \in \mathbb{N} \bigcup\{0\}, s \in \mathbb{C}$, we have

$$
C_{n, \lambda}^{(k)}=\sum_{l=0}^{n}\left(\begin{array}{l}
n \\
l
\end{array}\right) \sum_{r=0}^{l}\left(\begin{array}{l}
l \\
r
\end{array}\right) \sum_{m=0}^{r} \frac{(1)_{m+1, \lambda} S_{1, \lambda}(r+1, m+1)}{(r+1)(m+1)^{k-1}} C_{l-r, \lambda}^{*} \lambda^{n-l} B_{n-l}^{(n-l)} .
$$

From (2.1), we note that

$$
\begin{gathered}
\sum_{n=0}^{\infty} C_{n, \lambda}^{(k)}(1) \frac{t^{n}}{n !}=\frac{\operatorname{Ei}_{\mathrm{k}, \lambda}\left(\log _{\lambda}(1+\mathrm{t})\right)}{\log \left(1+\frac{1}{\lambda} \log (1+\lambda t)\right)}\left(1+\frac{1}{\lambda} \log (1+\lambda t)\right) \\
=\frac{\operatorname{Ei}_{\mathrm{k}, \lambda}\left(\log _{\lambda}(1+\mathrm{t})\right)}{\log \left(1+\frac{1}{\lambda} \log (1+\lambda t)\right)}+\frac{\operatorname{Ei}_{\mathrm{k}, \lambda}\left(\log _{\lambda}(1+\mathrm{t})\right) \frac{1}{\lambda} \log (1+\lambda \mathrm{t})}{\log \left(1+\frac{1}{\lambda} \log (1+\lambda t)\right)} \\
=\sum_{n=0}^{\infty} C_{n, \lambda}^{(k)} \frac{t^{n}}{n !}+t \frac{\operatorname{Ei}_{\mathrm{k}, \lambda}\left(\log _{\lambda}(1+\mathrm{t})\right)}{t} \frac{\frac{1}{\lambda} \log (1+\lambda t)}{\log \left(1+\frac{1}{\lambda} \log (1+\lambda t)\right)} \\
\sum_{n=1}^{\infty}\left[C_{n, \lambda}^{(k)}(1)-C_{n, \lambda}^{(k)}\right] \frac{t^{n}}{n !}=t \frac{\operatorname{Ei}_{\mathrm{k}, \lambda}\left(\log _{\lambda}(1+\mathrm{t})\right)}{t} \frac{\frac{1}{\lambda} \log (1+\lambda t)}{\log \left(1+\frac{1}{\lambda} \log (1+\lambda t)\right)} \\
=\left(\sum _ { m = 0 } ^ { \infty } \frac { ( 1 ) _ { m + 1 , \lambda } ( \operatorname { l o g } _ { \lambda } ( 1 + t ) ) ^ { m + 1 } ( m + 1 ) ! } { m ! ( m + 1 ) ^ { k } } \left(\sum_{l=0}^{\infty} \frac{(1)_{m, \lambda}\left(\log _{\lambda}(1+t)\right)^{m}}{(m+1) !}\left(\sum_{l=0}^{\infty} C_{l, \lambda}^{*} \frac{t^{l}}{l !}\right)\right.\right. \\
=\left(\sum_{r=0}^{\infty} \sum_{m=0}^{r} \frac{(1)_{m+1, \lambda} S_{1, \lambda}(r+1, m+1)}{(r+1)(m+1)^{k-1}} \frac{t^{r}}{r !}\right)\left(\sum_{l=0}^{\infty} C_{l, \lambda}^{*} \frac{t^{l}}{l !}\right) \\
\text { R.H.S }=\sum_{n=0}^{\infty}\left(\sum_{r=0}^{n}\left(\begin{array}{l}
n \\
r
\end{array}\right) \sum_{m=0}^{r} \frac{(1)_{m+1, \lambda} S_{1, \lambda}(r+1, m+1)}{(r+1)(m+1)^{k-1}} C_{n-r, \lambda}^{*}\right) \frac{t^{n}}{n !} .
\end{gathered}
$$

On the other hand,

$$
\begin{gathered}
\sum_{n=1}^{\infty}\left[C_{n, \lambda}^{(k)}(1)-C_{n, \lambda, 2}^{(k)}\right] \frac{t^{n-1}}{n !} \\
=\sum_{n=0}^{\infty}\left[\frac{C_{n+1, \lambda, 2}^{(k)}(1)-C_{n+1, \lambda, 2}^{(k)}}{n+1}\right] \frac{t^{n}}{n !} .
\end{gathered}
$$

Therefor, by (2.21) and (2.22), we obtain the following theorem.

Theorem 2.8. For $n \geq 0$, we have

$$
\frac{C_{n+1, \lambda}^{(k)}(1)-C_{n+1, \lambda}^{(k)}}{n+1}=\sum_{r=0}^{n}\left(\begin{array}{l}
n \\
r
\end{array}\right) \sum_{m=0}^{r} \frac{(1)_{m+1, \lambda} S_{1, \lambda}(r+1, m+1)}{(r+1)(m+1)^{k-1}} C_{n-r, \lambda}^{*} .
$$

\section{Type 2 degenerate unipoly-Cauchy polynomials of the second kind}

Let $p$ be any arithmetic function which is a real or complex valued function defined on the set of positive integers $\mathbb{N}$. Kim-Kim [11] defined the unipoly function attached to polynomials $p(x)$ by

$$
u_{k}(x \mid p)=\sum_{n=1}^{\infty} \frac{p(n)}{n^{k}} x^{n},(k \in \mathbb{Z})
$$

Moreover,

$$
u_{k}(x \mid 1)=\sum_{n=1}^{\infty} \frac{x^{n}}{n^{k}}=\operatorname{Li}_{k}(x),(\text { see }[5])
$$


is the ordinary polylogaritm function.

In this paper, we define the degenerate unipoly function attached to polynomials $p(x)$ as follows:

$$
u_{k, \lambda}(x \mid p)=\sum_{i=1}^{\infty} p(i) \frac{(1)_{i, \lambda} x^{i}}{i^{k}}
$$

It is worthy to note that

$$
u_{k, \lambda}\left(x \mid \frac{1}{\Gamma}\right)=\operatorname{Ei}_{k, \lambda}(x)
$$

is the degenerate modified polyexponential function.

By using (3.3), we define the type 2 degenerate unipoly-Cauchy polynomials of the second kind as follows:

$$
\frac{u_{k, \lambda}\left(\log _{\lambda}(1+t) \mid p\right)}{\log \left(1+\frac{1}{\lambda} \log (1+\lambda t)\right)}\left(1+\frac{1}{\lambda} \log (1+\lambda t)\right)^{\frac{x}{\lambda}}=\sum_{n=0}^{\infty} C_{n, \lambda, p}^{(k)}(x) \frac{t^{n}}{n !} .
$$

In the case when $x=0, C_{n, \lambda, p}^{(k)}=C_{n, \lambda, p}^{(k)}(0)$ are called the type degenerate unipolyCauchy numbers of the second kind. Let us take $p(n)=\frac{1}{\Gamma \lambda}$. Then we have

$$
\begin{gathered}
\sum_{n=0}^{\infty} C_{n, \lambda, \frac{1}{\Gamma}}^{(k)}(x) \frac{t^{n}}{n !}=\frac{u_{k, \lambda}\left(\log _{\lambda}(1+t) \mid \frac{1}{\Gamma} p\right)}{\log \left(1+\frac{1}{\lambda} \log (1+\lambda t)\right)}\left(1+\frac{1}{\lambda} \log (1+\lambda t)\right)^{\frac{x}{\lambda}} \\
=\frac{1}{\log \left(1+\frac{1}{\lambda} \log (1+\lambda t)\right)} \sum_{m=1}^{\infty} \frac{(\log (1+t))^{m}}{m^{k}(m+1) !}\left(1+\frac{1}{\lambda} \log (1+\lambda t)\right)^{\frac{x}{\lambda}} \\
=\frac{\operatorname{Ei}_{\mathrm{k}, \lambda}\left(\log _{\lambda}(1+t)\right)}{\log \left(1+\frac{1}{\lambda} \log (1+\lambda t)\right)}\left(1+\frac{1}{\lambda} \log (1+\lambda t)\right)^{\frac{x}{\lambda}} \\
=\sum_{n=0}^{\infty} C_{n, \lambda}^{(k)}(x) \frac{t^{n}}{n !}
\end{gathered}
$$

Thus, by (3.6), we have the following theorem.

Theorem 3.1. Let $n \geq 0$ and $k \in \mathbb{Z}$, and $\Gamma n$ be a Gamma function. Then, we have

$$
C_{n, \lambda, \frac{1}{\Gamma}}^{(k)}(x)=C_{n, \lambda}^{(k)}(x) .
$$

From (3.5), we get

$$
\begin{gathered}
\sum_{n=0}^{\infty} C_{n, \lambda, p}^{(k)} \frac{t^{n}}{n !}=\frac{u_{k, \lambda}\left(\log _{\lambda}(1+t) \mid p\right)}{\log \left(1+\frac{1}{\lambda} \log (1+\lambda t)\right)} \\
=\frac{1}{\log \left(1+\frac{1}{\lambda} \log (1+\lambda t)\right)} \sum_{m=1}^{\infty} \frac{p(m)(1)_{m, \lambda}}{m^{k}}\left(\log _{\lambda}(1+t)\right)^{m} \\
=\frac{1}{\log \left(1+\frac{1}{\lambda} \log (1+\lambda t)\right)} \sum_{m=0}^{\infty} \frac{p(m+1)(1)_{m+1, \lambda}(m+1) !}{(m+1)^{k}} \sum_{l=m+1}^{\infty} S_{1, \lambda}(m+1, l) \frac{t^{l}}{l !} \\
=\left(\sum_{j=0}^{\infty} C_{j, \lambda} \frac{t^{j}}{j !}\right)\left(\sum_{m=0}^{\infty} \sum_{l=0}^{m} \frac{p(m+1)(1)_{m+1, \lambda}(m+1) !}{(m+1)^{k}} S_{1, \lambda}(m+1, l) \frac{t^{l}}{l !}\right) \\
=\sum_{n=0}^{\infty}\left(\sum_{l=0}^{\infty} \sum_{m=0}^{l}\left(\begin{array}{l}
n \\
l
\end{array}\right) \frac{p(m+1)(1)_{m+1, \lambda}(m+1) ! S_{1, \lambda}(m+1, l+1) C_{n-l, \lambda}}{(m+1)^{k}(l+1)}\right) \frac{t^{n}}{n !} .
\end{gathered}
$$


Therefore, by comparing the coefficients on both sides of (3.8), we obtain the following theorem.

Theorem 3.2. Let $n \in \mathbb{N}$ and $k \in \mathbb{Z}$. Then we have

$$
C_{n, \lambda, p}^{(k)}=\sum_{l=0}^{\infty} \sum_{m=0}^{l}\left(\begin{array}{l}
n \\
l
\end{array}\right) \frac{p(m+1)(1)_{m+1, \lambda}(m+1) ! S_{1, \lambda}(m+1, l+1) C_{n-l, \lambda}}{(m+1)^{k}(l+1)} .
$$

In particular,

$$
C_{n, \lambda, \frac{1}{\Gamma}}^{(k)}=C_{n, \lambda}^{(k)}=\sum_{l=0}^{\infty} \sum_{m=0}^{l}\left(\begin{array}{l}
n \\
l
\end{array}\right) \frac{S_{1, \lambda}(m+1, l+1) C_{n-l, \lambda, 2}}{(m+1)^{k-1}(l+1)} .
$$

From (3.5), we observe that

$$
\begin{gathered}
\sum_{n=0}^{\infty} C_{n, \lambda}^{(k, p)}(x) \frac{t^{n}}{n !}=\frac{u_{k, \lambda}\left(\log _{\lambda}(1+t) \mid p\right)}{\log \left(1+\frac{1}{\lambda} \log (1+\lambda t)\right)}\left(1+\frac{1}{\lambda} \log (1+\lambda t)\right)^{\frac{x}{\lambda}} \\
=\frac{u_{k, \lambda}\left(\log _{\lambda}(1+t) \mid p\right)}{\log \left(1+\frac{1}{\lambda} \log (1+\lambda t)\right)} \sum_{m=0}^{\infty}\left(\begin{array}{c}
\frac{x}{\lambda} \\
m
\end{array}\right)\left(\frac{1}{\lambda} \log (1+\lambda t)\right)^{m} \\
=\left(\sum_{l=0}^{\infty} C_{l, \lambda, p}^{(k)} \frac{t^{l}}{l !}\right)\left(\sum_{m=0}^{\infty}(x)_{m, \lambda} \lambda^{-2 m} \sum_{s=m}^{\infty} S_{1}(s, m) \frac{t^{s}}{s !}\right) \\
=\left(\sum_{l=0}^{\infty} C_{l, \lambda, p}^{(k)} \frac{t^{l}}{l !}\right)\left(\sum_{s=0}^{\infty} \sum_{m=0}^{s}(x)_{m, \lambda} \lambda^{-2 m} S_{1}(s, m) \frac{t^{s}}{s !}\right) \\
\text { L.H.S }=\sum_{n=0}^{\infty}\left(\sum_{l=0}^{n} \sum_{m=0}^{n-l} C_{l, \lambda, p}^{(k)}(x)_{m, \lambda} \lambda^{-2 m} S_{1}(n-l, m)\right) \frac{t^{n}}{n !} .
\end{gathered}
$$

From (3.11), we obtain the following theorem.

Theorem 3.3. Let $n \geq 0$ and $k \in \mathbb{Z}$. Then we have

$$
C_{n, \lambda, p}^{(k)}(x)=\sum_{l=0}^{n} \sum_{m=0}^{n-l} C_{l, \lambda, p}^{(k)}(x)_{m, \lambda} \lambda^{-2 m} S_{1}(n-l, m)
$$

From (3.5), we observe that

$$
\begin{gathered}
\sum_{n=0}^{\infty} C_{n, \lambda, p}^{(k)} \frac{t^{n}}{n !}=\frac{u_{k, \lambda}\left(\log _{\lambda}(1+t) \mid p\right)}{\log \left(1+\frac{1}{\lambda} \log (1+\lambda t)\right)} \\
=\frac{1}{\log \left(1+\frac{1}{\lambda} \log (1+\lambda t)\right)} \sum_{m=0}^{\infty} \frac{p(m+1)(1)_{m+1, \lambda} m !}{(m+1)^{k} m !}\left(\log _{\lambda}(1+t)\right)^{m+1} \\
=\frac{\log _{\lambda}(1+t)}{\log \left(1+\frac{1}{\lambda} \log (1+\lambda t)\right)} \sum_{m=0}^{\infty} \frac{p(m+1)(1)_{m+1, \lambda} m !}{(m+1)^{k} m !}\left(\log _{\lambda}(1+t)\right)^{m} \\
=\frac{\log _{\lambda}(1+t)}{t} \frac{t}{\log \left(1+\frac{1}{\lambda} \log (1+\lambda t)\right)} \sum_{m=0}^{\infty} \frac{p(m+1)(1)_{m+1, \lambda} m !}{(m+1)^{k}} \sum_{l=m}^{\infty} S_{1, \lambda}(l, m) \frac{t^{l}}{l !} \\
=\left(\sum_{s=0}^{\infty} D_{s, \lambda} \frac{t^{s}}{s !}\right)\left(\sum_{a=0}^{\infty} C_{a, \lambda} \frac{t^{a}}{a !}\right)\left(\sum_{l=0}^{\infty} \sum_{m=0}^{n} \frac{p(m+1)(1)_{m+1, \lambda} m !}{(m+1)^{k}} S_{1, \lambda}(l, m) \frac{t^{l}}{l !}\right)
\end{gathered}
$$




$$
\begin{gathered}
=\left(\sum_{b=0}^{\infty} \sum_{a=0}^{b}\left(\begin{array}{l}
b \\
a
\end{array}\right) D_{b-a, \lambda} C_{a, \lambda} \frac{t^{b}}{b !}\right)\left(\sum_{l=0}^{\infty} \sum_{m=0}^{n} \frac{p(m+1)(1)_{m+1, \lambda} m !}{(m+1)^{k}} S_{1, \lambda}(l, m) \frac{t^{l}}{l !}\right) \\
\text { L.H.S }=\sum_{n=0}^{\infty}\left(\sum_{l=0}^{n} \sum_{a=0}^{n-l} \sum_{m=0}^{l}\left(\begin{array}{c}
n \\
l
\end{array}\right) D_{n-l-a, \lambda} C_{a, \lambda} \frac{p(m+1)(1)_{m+1, \lambda} m !}{(m+1)^{k}} S_{1, \lambda}(l, m)\right) \frac{t^{n}}{n !} .
\end{gathered}
$$

By comparing coefficients on both sides of (3.13), we obtain the following theorem.

Theorem 3.4. Let $n \geq 0$ and $k \in \mathbb{Z}$. Then we have

$$
C_{n, \lambda, p}^{(k)}=\sum_{l=0}^{n} \sum_{a=0}^{n-l} \sum_{m=0}^{l}\left(\begin{array}{l}
n \\
l
\end{array}\right) D_{n-l-a, \lambda} C_{a, \lambda} \frac{p(m+1)(1)_{m+1, \lambda} m !}{(m+1)^{k}} S_{1, \lambda}(l, m) .
$$

\section{Conclusions}

In 2020 Kim-Kim considered the type degenerate poly-Bernoulli polynomials by making use of the modified polylogarithm functions and Kim [14] introduced the degenerate Cauchy numbers of the second kind. By using these functions and polynomials, we defined the type 2 degenerate poly-Cauchy polynomials of the second kind and obtained some identities of the degenerate poly-Cauchy numbers of the second kind in Theorems 2.1 and 2.2. In particular, we obtained an identity of the degenerate polyCauchy polynomials of the second kind in Theorem 2.3. Furthermore, by using the unipoly functions, we defined the degenerate unipoly-Cauchy polynomials of the second kind(Eq. (3.5)) and obtained some properties of the degenerate unipoly-Cauchy numbers of the second kind(Theorems 3.1, and 3.3). Finally, we obtained an identity of the degenerate unipoly-Cauchy polynomials of the second kind in Theorem 3.3 and gave the identity indicating the relationship of the degenerate unipoly-Cauchy numbers of the second kind and the Daehee numbers and degenerate Cauchy numbers of the second kind in Theorem 3.4.

Author Contributions: All authors contributed equally to the manuscript and typed, read, and approved final manuscript.

Conflict of Interest: The authors declare no conflict of interest.

Funding: None.

Acknowledgements: None.

\section{References}

[1] L. Catlitz, A degenerate Staudt-Clausen theorem. Arch. Math. (Basel).7(1967), 28-33.

[2] L. Catlitz, Degenerate Stirling, Bernoulli and Eulerian numbers. Util. Math. 15 (1979), 51-88.

[3] B. S. Borisov, The p-binomial transform Cauchy numbers and figurate numbers, Proc. Jangjeon Math. Soc. 19(4),(2016), 631-644. 
[4] S.-K. Chung, G.-W. Jang, D. S. Kim and J. Kwon, Some identities of the type 2 degenerate Bernoulli and Euler numbers, Adv. Stud. Contemp. Math. (Kyungshang) 29(4),(2019), 613-632.

[5] L.C. Jang, W. Kim, H.-I. Kwon, T. Kim, On degenerate Daehee polynomials and numbers of the third kind, J. Comput. Appl. Math. 364, (2020), 112343, 9 pp.

[6] D.S. Kim, T. Kim, A note on polyexponential and unipoly functions, Russ. J. Math. Phys. 26(1),(2019), 40-49.

[7] D. S. Kim, T. Kim, On sums of finite products of balancing polynomials, Comput. Appl. Math. 377, (2020), 112913.

[8] T. Kim, D. S. Kim, H. Y. Kim, L.C.Jang, Degenerate poly-Bernoulli numbers and polynomials, Informatica , 31(3),(2020), 2-8.

[9] T. Kim, D. S. Kim, D. V. Dolgy, J. Kwon, Some identities on generalized degenerate Genocchi and Euler numbers, Informatica, 31(4),(2020), 42-51.

[10] T. Kim, D. S. Kim, J. Kwon, H. Lee, A note on degenerate gamma random variables, Revista de Educacion 388(4),(2020), 39-44.

[11] T. Kim, D. S. Kim, Degenerate polyexponential functions and degenerate Bell polynomials, J. Math. Anal. Appl. 487(2),(2020),124017.

[12] T. Kim, Degenerate Cauchy numbers and polynomials of the second kind, Adv. Stud. Contemp. Math. (Kyungshang) 27,(2017), 441-449.

[13] T. Kim , On degenerate Cauchy numbers and polynomials, Proc. Jangjeon Math. Soc. 18(3),(2015), 307-312.

[14] M. Kaneko, M. "poly-Bernoulli numbers". J. Théor Nombres Bordeaux. 1997, 9(1), 221-228.

[15] T. Kim, A note on degenerate Stirling numbers of the second kind Proc. Jangjeon Math. Soc.. 21(4)(2018), 589-598.

[16] T. Kim, D. S. Kim, H.-Y. Kim, J. Kwon, A new type degenerate Daehee numbers and polynomials, 2020, arXiv:2004.08743v1 [math.NT] 19 Apr 2020.

[17] T. Kim, D. S. Kim, L. C. Jang, H.-Y. Kim, On type 2 degenerate Bernoulli and Euler polynomials of complex variable. Adv. Difference Equ. 2019, Paper No.490, 15pp.

[18] T. Kim, W. A. Khan, S. K. Sharma, M. Ghayasuddin, A note on parametric kinds of the degenerate poly-Bernoulli and poly-Genocchi polynomials. Symmetry. 12(4)(2020), Article ID 614.

[19] W. A. Khan and M. Ahmad, Partially degenerate poly-Bernoulli polynomials associated with Hermite polynomials, Adv. Stud. Contemp. Math. (Kyungshang) 28(3),(2018), 487-496.

[20] W. A. Khan, A new class of degenerate Frobenius-Euler-Hermite polynomials, Adv. Stud. Contemp. Math. (Kyungshang) 28(4),(2018), 567-576.

[21] T. Komatsu, Higher-order convolution identities for Cauchy numbers of the second kind,Proc. Jangjeon Math. Soc. 18(3),(2015), 369-383.

[22] S.-S. Pyo, Degenerate Cauchy numbers and polynomials of the fourth kind, Adv. Stud. Contemp. Math. (Kyungshang) 28(1),(2018), 127-138.

[23] S. -S. Pyo, T. Kim, S. -H. Rim, Degenerate Cauchy numbers of the third kind, J. Ineq. Appl. 2018:32,(2018), 12pp.

[24] S. Roman, The umbral calculus, Pure and Applied Mathematics, 111. Academic Press, Inc. [Harcourt Brace Jovanovich, Publishers], New York, 1984. $\mathrm{x}+193$ pp. ISBN: 0-12-594380-6. 